\title{
The Method of Checking Equations for Energy Resources Flows Data Validating
}

\author{
Vladislav O. Samoylenko ${ }^{1}$, Andrew V. Pazderin ${ }^{1}$ \\ ${ }^{1}$ Department of Automated Electrical Systems \\ Boris Yeltzin Ural Federal University \\ Mira, 19, 620002, Ekaterinburg (Russia) \\ Phone/Fax number: +007912 2675288, e-mail: vedmed@e1.ru, pav@daes.ustu.ru
}

\begin{abstract}
The development of energy resources flows verifying methods is aimed primarily at automatic energy accounting systems improvement. Creation of the hi-technology energy resources metering and accounting systems supports the practical application of the total energy efficiency concept and enables the financial mutual settlements between the parties to be improved. The paper presents an advanced mathematical method of checking equations (CE) for monitoring the validity of metering data in the energy transportation networks. The analysis of CE mismatches allows all the measurements of any energy resource flows to be divided into four groups: true, false, uncertain and untestable (critical). The method proposed in the paper gives an opportunity to detect bad data and its source. Also, it provides for a bad data filtering at the stage of data acquisition.
\end{abstract}

\section{Key words}

Data validation, energy efficiency, electric energy metering, checking equations, state estimation.

\section{Introduction}

The ongoing implementation of microgrids and distributed generation requires the technologies for direct monitoring of the energy efficiency. There are a number of methods making the precious electrical energy accounting or heat energy accounting possible. Nevertheless, due to the widespread penetration of micro-cogeneration a complex energy resources flows data validating method is needed.

The development of energy resources flows verifying methods is aimed primarily at automatic energy accounting systems improvement. Creation of the hi-technology energy resources metering and accounting systems supports the practical application of the total energy efficiency concept and enables the financial mutual settlements between the parties to be improved. Currently, advanced automated metering systems for energy resources control and accounting (AMR) are being created.

The main framework for most types of energy transporting is a developed network infrastructure. Pipelines, heating mains and networks as elements of this infrastructure make the oil and gas industry as well as the heat and electrical power engineering industries similar. The interrela- tions in an energy transporting network are shown in Figure 1.

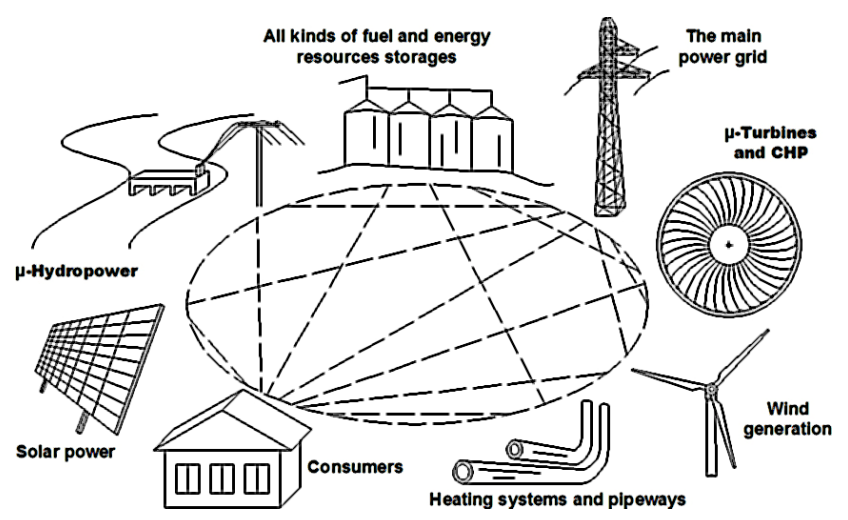

Fig. 1. The interrelations of the different components of an energy transporting network

This results in the mathematical models used in research in these fields being also similar, while the physical essence of the processes remains different. The system of the energy resources transportation can be represented as a graph, with nodes being producers or consumers and branches being the mains (transmission lines, pipelines or heat mains).

AMR is expected to be able to recognize invalid data and restore the information lost, to detect The paper presents method of checking equations for monitoring the validity of metering data in the energy transportation networks. The method is based on the general energy resources flows data validating [1] which has been inspired by the state estimation theory and serves as a practical application of energy flow problem [2].

The method is aiming primarily at the AMR software development for the purposes of energy efficiency improvement and mutual financial settlements to be kept.

\section{Theoretical background}

In an arbitrary network including a number of nodes and branches, there are nodal injections of energy from producers (electrical energy, a heat-transfer agent, a gas or 
solid fuel flow), the energy resource consumption in demand nodes, overflows in branches (i.e. power lines or pipelines) and losses (Figure 2).

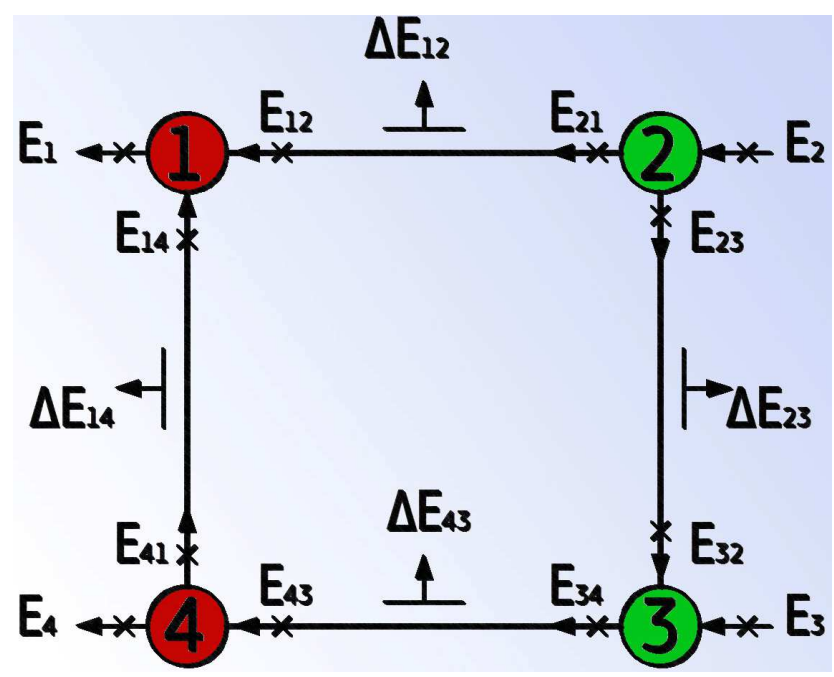

Fig. 2. A model of energy transporting network

The energy resource losses in branches depend upon the specific features of a particular type of the energy resource and a physical principle of the transfer process.

In Figure 1, the symbol " $X$ " denotes the presence of a metering system of an energy resource and the place of its location.

There is an energy resource flows balance at any moment. The equations of the balance can be represented as follows:

$$
\sum_{j \in \omega_{i}} E_{i j}+E_{i}=0, \quad i=1,2, \ldots, N,
$$

where $E_{i}$ is a nodal injection or consumption in node $i$; $E_{i j}$ is energy resource flows in branches connected to node $i ; \omega_{i}$ is a set of nodes, adjacent to $i$ node; $N$ is the number of nodes.

Similarly, the balance equations for the branches are:

$$
E_{i j}+E_{j i}-\Delta E_{i j}=0, \quad i=1,2, \ldots, M,
$$

where $E_{i j}$ is flows at the beginning of the branches; $E_{j i}$ is flows at the end of the branches; $\Delta E_{i j}$ is losses in the branches; $M$ is the number of branches.

Any measurement system has a certain error, so the balances based on the measurements are not satisfied in the nodes. Also, the values of measured flows are not identical to the caclulated ones. The energy measurement coordination is aimed at minimizing the sum of squares of relative measurements errors:

$$
F=\sum_{i=1}^{K} r_{i}\left(\frac{E_{i}^{\text {meas }}-E_{i}^{\text {calc }}}{E_{i}^{\text {meas }}}\right)^{2} \rightarrow \min ,
$$

where $E_{i}^{\text {meas }}$ is measured values; $E_{i}^{\text {calc }}$ is calculated values; $r_{i}$ is weight factors which are inversely proportional to the accuracy $\delta_{i}$ of the measurement system:

$$
r_{i}=\frac{1}{\left(\delta_{i}\right)^{2}}
$$

Energy measurement coordination is a typical nonlinear programming problem to be solved by appropriate methods [3].

As a matrix, the system of equations for all the measurements can be represented by:

$$
\mathbf{H} \cdot \mathbf{E}_{\mathbf{i}}^{\text {calc }}=\mathbf{E}_{\mathbf{i}}^{\text {meas }},
$$

where $\mathbf{H}$ is an observability matrix. $\mathbf{E}_{\mathbf{i}}^{\text {meas }}$ dimension is set by $\mathrm{K}$, and $\mathbf{E}_{\mathbf{i}}^{\text {calc }}$ dimension is set by $\mathbf{M}$. Depending on the dimensions matching, there can be distinguished three different cases.

The first case deals with $K<M$, matrix $\mathbf{H}$ being rectangular, there is an infinite set of uncertain solutions. The lack of measurement data results in energy resource flows distribution being impossible to obtain.

The second case deals with $K=M$, matrix $\mathbf{H}$ being square, there is the only solution. Nevertheless, it is impossible to recognize the measurement errors and mitigate their impact.

In the case of $K>M$, matrix $\mathbf{H}$ is rectangular, the solution is determined in terms of the energy flow problem. The measurement data is redundant. The solution of the redefined equation set (5) can be obtained by transforming it into a regular form equation set:

$$
\left(\mathbf{H}^{\mathrm{T}} \cdot \mathbf{R}^{-1} \cdot \mathbf{H}\right) \cdot \mathbf{E}_{\mathrm{b}}^{\mathrm{calc}}=\mathbf{H}^{\mathrm{T}} \cdot \mathbf{R}^{-1} \cdot \mathbf{E}^{\text {meas }},
$$

where $\mathbf{R}^{-1}$ is a diagonal weight ratio matrix, the dimensions of which are equal to the number of measurements $K$. According to (4), the diagonal elements of the given matrix are defined as follows:

$$
r_{i}=\frac{1}{\left(\delta_{i} \cdot E_{i}^{\text {meas }}\right)^{2}},
$$

The conditions of observability, connected with topology of an energy transportation network and placing of metering systems, are defined as topological observability [2]. In the state estimation theory it is proved that the observability of the scheme is to be ensured by the number of measurement systems being not less than the number of branches $M$ in the scheme.

\section{The checking equations method}

Checking equations technique for state estimation applications is one of the most promising nowadays. The analysis of CE mismatches allows all the measurements to be divided into four groups: true, false, uncertain and untestable (critical).

In general algorithm for checking equations obtaining $\mathrm{CE}$ contain only measured variables. Provided by necessary measurements for all elements of the network, the set of linearly independent $\mathrm{CE}$ will coincide with the energy balance equations for nodes (1) and for branches (2).

Putting actual measurements and rated losses into the left parts of CE will lead to mismatches appearance in the right part of the equations instead of zeroes. The matrix algorithm of checking equations obtaining is shown in Figure 3. 


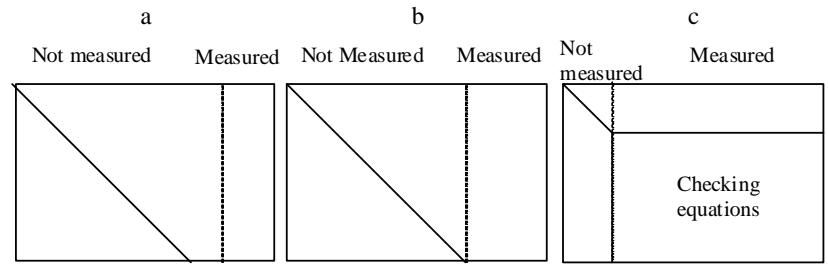

Fig. 3. The matrix algorithm of checking equations obtaining

The analysis of these mismatches makes possible to detect bad metering data. The main condition of the energy efficiency check will be:

$$
\operatorname{Im} b_{\text {real }} \leq \operatorname{Im} b_{\max }(1)
$$

Real imbalance $\operatorname{Im} b_{\text {real }}$ is defined as a sum of all the flows:

$$
\operatorname{Im} b_{\text {real }}=\frac{E_{\text {inf }}-E_{\text {eff }}-\Delta E_{\text {tech }}}{E_{\text {inf }}} 100 \%,
$$

where $E_{\text {inf }}$ is influent energy flows; $E_{\text {eff }}$ is effluent energy flows; $\Delta E_{\text {tech }}$ is losses.

Maximum imbalance $I m b_{\max }$ is defined as:

$$
\delta_{\Sigma}=\sqrt{\sum_{i}\left(\delta_{i} \cdot E_{i}\right)^{2}}
$$

where $\delta_{i}$ is an relative error of an energy meter installed at point $i ; E_{i}$ is energy measured by meter at point $i$.

\section{An example}

An example of the checking equations use is shown in Figure 4.

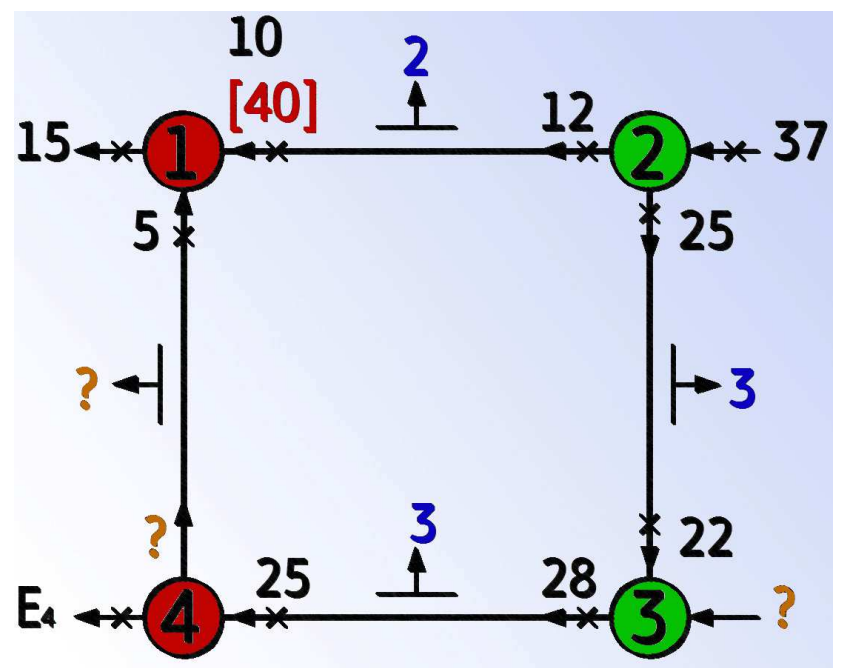

Fig. 4 - Energy flows data (in MWh) for energy transporting network

In Figure 3 normal measurements are colored in black and calculated values are colored in blue. Lost or damaged data are colored in orange. Bad data $E_{12}$ are colored in red. The system of energy flows equations will be follow:

$$
\begin{aligned}
& E_{1}+E_{12}+E_{14}=0 \\
& E_{2}+E_{21}+E_{23}=0 \\
& \underline{E_{3}}+E_{32}+E_{34}=0 \\
& E_{4}+\underline{E_{41}}+E_{43}=0 \\
& E_{12}+E_{21}+\Delta E_{12 \text { tech }}=0 \\
& E_{23}+E_{32}+\Delta E_{23 \text { tech }}=0 \\
& E_{14}+\underline{E_{41}}+\Delta E_{14 \text { tech }}=0 \\
& E_{43}+E_{34}+\Delta E_{34 \text { tech }}=0
\end{aligned}
$$

Here the unknown, lost or damaged data are underlined. The modified system of checking equations including the measured and calculated values only can be presented as:

$$
\begin{aligned}
& E_{1}+E_{12}+E_{14}=0 \\
& E_{2}+E_{21}+E_{23}=0 \\
& E_{4}+E_{43}-E_{14}-\Delta E_{14 \text { tech }}=0 \\
& E_{12}+E_{21}+\Delta E_{12 \text { tech }}=0 \\
& E_{23}+E_{32}+\Delta E_{23 \text { tech }}=0 \\
& E_{43}+E_{34}+\Delta E_{34 \text { tech }}=0
\end{aligned}
$$

As it can be seen from (12), it is possible to avoid usage of lost or damaged data eliminating corresponding variables. This important advantage of the method can be easily implemented in automated measurement systems using the Gaussian elimination.

Also it is possible to detect the source of bad data and to make a data request. If repeating invalid data are obtained, so, it is possible to establish a defective meter or measurement complex.

The first step is the maximum imbalance calculation for each control equation in accordance with (10). If all the measurement complexes' accuracies are set by $2 \%$, for the first checking equation the maximum imbalance will be:

$$
\begin{aligned}
& \delta_{1}=\sqrt{\sum_{i}\left(\delta_{i} \cdot E_{i}\right)^{2}}= \\
& =\sqrt{(0,02 \cdot(-15))^{2}+(0,02 \cdot 10)^{2}+(0,02 \cdot 5)^{2}}=0,374,
\end{aligned}
$$

Maximum imbalances for the other checking equations in the system can be calculated in a similar way. If bad data are obtained $\left(E_{12}=40\right.$ instead of $\left.E_{12}=10\right)$, the result of energy resources flows imbalances calculation will be following:

$$
\begin{aligned}
& -15+40+5=30>0,374 \\
& 37-12-25=0<0,925 \\
& -19+25-6-5=0<0,647 \\
& -12+40+2=30>0,140 \\
& -25+22+3=0<0,730 \\
& -28+25+3=0<0,808
\end{aligned}
$$

Using serial checks for the variables it is possible to find a bad data source. The balances for the first and the fourth equations (14) are not met. The system of equations for $E_{14}$ check: 


$$
\begin{aligned}
& E_{1}+E_{12}+E_{4}+E_{43}-E_{14 \text { tech }}=0 \\
& E_{2}+E_{21}+E_{23}=0 \\
& E_{12}+E_{21}+\Delta E_{12 \text { tech }}=0 \\
& E_{23}+E_{32}+\Delta E_{23 \text { tech }}=0 \\
& E_{43}+E_{34}+\Delta E_{34 \text { tech }}=0
\end{aligned}
$$

The values of variables will be:

$$
\begin{aligned}
& -15+40-19+25-1=30 \\
& 37-12-25=0 \\
& -12+40+2=30 \\
& -25+22+3=0 \\
& -28+25+3=0
\end{aligned}
$$

In spite of the fact that $E_{14}$ variable was eliminated, the equations contains bad data that are the reasons of the balances not met. The most likely source of incorrect data is $E_{12}$ due to the fact that it is included in both equations.

$$
\begin{aligned}
& W_{1}-W_{21}-\Delta W_{12 \text { тех }}+W_{14}=0 \\
& W_{2}+W_{21}+W_{23}=0 \\
& W_{4}+W_{43}-W_{14}-\Delta W_{14 \text { тех }}=0 \\
& W_{23}+W_{32}+\Delta W_{23 \text { тех }}=0 \\
& W_{43}+W_{34}+\Delta W_{34 \text { тех }}=0
\end{aligned}
$$

The values of variables will be:

$$
\begin{aligned}
& -15+12+2+1=0 \\
& 37-12-25=0 \\
& -19+25-5-1=0 \\
& -25+22+3=0 \\
& -28+25+3=0
\end{aligned}
$$

By $E_{12}$ elimination it is possible to meet the balances, so these data are invalid.

Anyway, the source of invalid data, i.e. defective meter or data transfer failure, will be detected. The checking equations technique can be improved to detect a number of wrong measurements which is more than one.

\section{Conclusion}

Within the frame of a single process, the CE technique gives the possibility to analyze observability, detect criti- cal measurements and groups of interrelated abundant measurements. In groups of redundant measurements, it is possible to find substituting values for the measurements which were rejected. One of the most important conclusions of this work deals with the necessity to build up information-redundant energy accounting systems. This is of special importance for metering stations of the commercial accounting. These stations contribute to the financial mutual settlements between the energy exchange participants.

The method proposed in the paper gives an opportunity to implement a number of useful functions of prospective metering systems.

1. To improve the reliability of measurements of energy (electricity, gas, oil, heat-transfer), the transportation of which is carried out by the network infrastructure. As a result, a unified automated measuring system for controlling and accounting energy resources will be created and the system components responsible for making calculations will be unified.

2. To detect a bad data, ensuring the possibility of reinquiring as well as identifying the source of false information and signaling a malfunction in the system; to assess the level of commercial losses.

3. To improve AMR software of complex energy resources accounting systems.

3. To improve the level of an energy efficiency in general. The method presented can form the framework of the next-generation high-tech systems for measuring and controlling energy resources. Taking into account the development of the production infrastructure and increasing attention being paid to energy efficiency, the method is very promising. The checking equations technique can be improved to detect a number of wrong measurements which is more than one.

\section{References}

[1] V.O. Samoylenko, A.V. Pazderin «A Mathematical Method of Energy Resources Flows Data Validating Using the State Estimation Theory» // Proceedings International Conference on Renewable Energies \& Power Quality, No. 10, Santiago de Compostela, Spain, 2012.

[2] Solution of energy flow problem using state estimation technique. 35th Annual Conference of IEEE Industrial Electronics Society, Porto, Portugal, 2009, p.1750-1755.

[3] A.V. Pazderin, E.A. Plesnyaev «Analysis of the Computation Techniques for Energy Flow Problem Solving» // Proceedings IEEE Conference «Computer as a Tool» (EURO 2005), Belgrade, Serbia \& Montenegro, 2005. pp. 589-593. 